Supplement of Hydrol. Earth Syst. Sci., 20, 3441-3454, 2016

http://www.hydrol-earth-syst-sci.net/20/3441/2016/

doi:10.5194/hess-20-3441-2016-supplement

(C) Author(s) 2016. CC Attribution 3.0 License.

(c) (1)

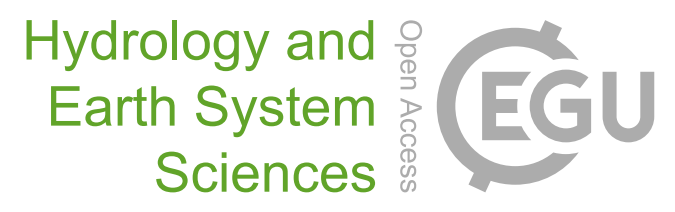

Supplement of

\title{
A thermodynamic formulation of root water uptake
}

Anke Hildebrandt et al.

Correspondence to: Anke Hildebrandt (anke.hildebrandt@uni-jena.de)

The copyright of individual parts of the supplement might differ from the CC-BY 3.0 licence. 
This supplement contains results of additional model scenarios in order to illustrate that the results presented in the main text are representative of a wider range of situations, namely transient boundary conditions and different soil textures.

First, the thermodynamic diagnostic can be applied for situations where water uptake changes at the diurnal scale, although we only present steady state scenarios in the main text. The calculated water and energy fluxes of the transient scenario show similar behavior as presented in the main text. We show them here for completeness (Fig S01 and S02).

Additionally, soil properties affect the break down of the exported energy into its components. In the main text, we present results for a sandy loam, that is a medium textured soil with average water retention. In the supplement, we add results for two more soil textures, located at more extreme ends of the soil water retention spectrum: A sand with very low water retention and a clay with very high water retention (Rawls et al., 1993, see Table $\mathrm{SO} 1$ for soil parameters and Figure $\mathrm{S03}$ for plots). The following figures give the result of the hydrologic model and derived thermodynamic fluxes for sand (Fig. S04, S05) and clay (S06 and S07).

\section{Tables}

Table S01: Parameters of the van Genuchten (van Genuchten, 1980) Equation applied in the main text (Sandy loam) and for the additional simulations performed for water retention curves of soils representative of sand and clay (Rawls et al. 1993). The corresponding soil water retention curves are plotted in Figure S03. Sandy loam is the soil type used in the main text.

\begin{tabular}{llll}
\hline & Sand & Sandy loam & Clay \\
\hline $\mathrm{n},-$ & 1.694 & 1.378 & 1.165 \\
$\alpha, \mathrm{cm}^{-1}$ & 0.138 & 0.068 & 0.027 \\
$\theta_{\min ,-}$ & 0.022 & 0.070 & 0.232 \\
$\theta_{\mathrm{r},-}$ & 0.020 & 0.041 & 0.090 \\
$\theta_{\mathrm{s},-}$ & 0.437 & 0.453 & 0.475 \\
\hline
\end{tabular}


Figures
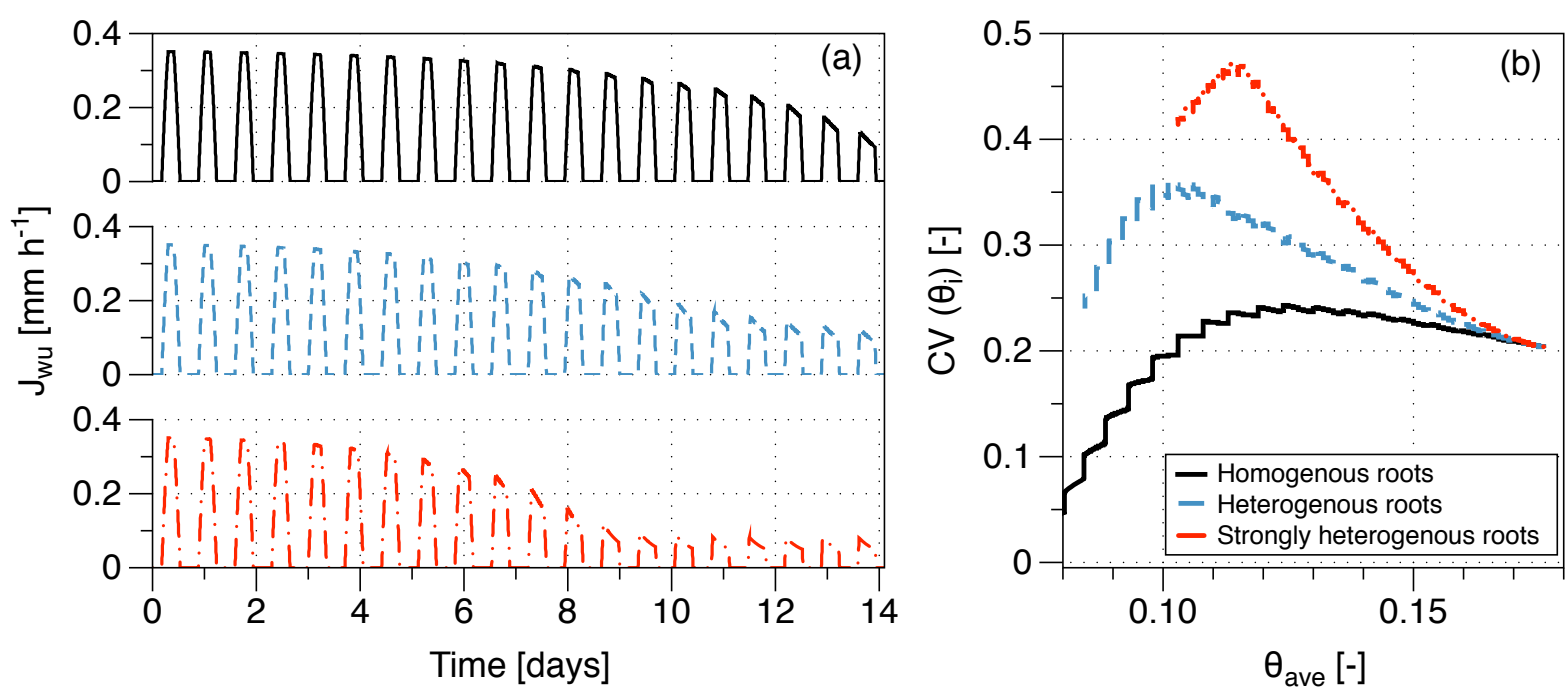

Fig. S01: Evolution of a distributed, transient root water uptake model. Total potential daily transpiration is $3 \mathrm{~mm} \mathrm{~d}^{-1}$. Root water uptake follows potential transpiration, unless root collar potential drops below the permanent wilting point $(-150 \mathrm{~m})$. (a) Evolution of the calculated root water uptake, (b) Coefficient of variation of water content in the three compartments.
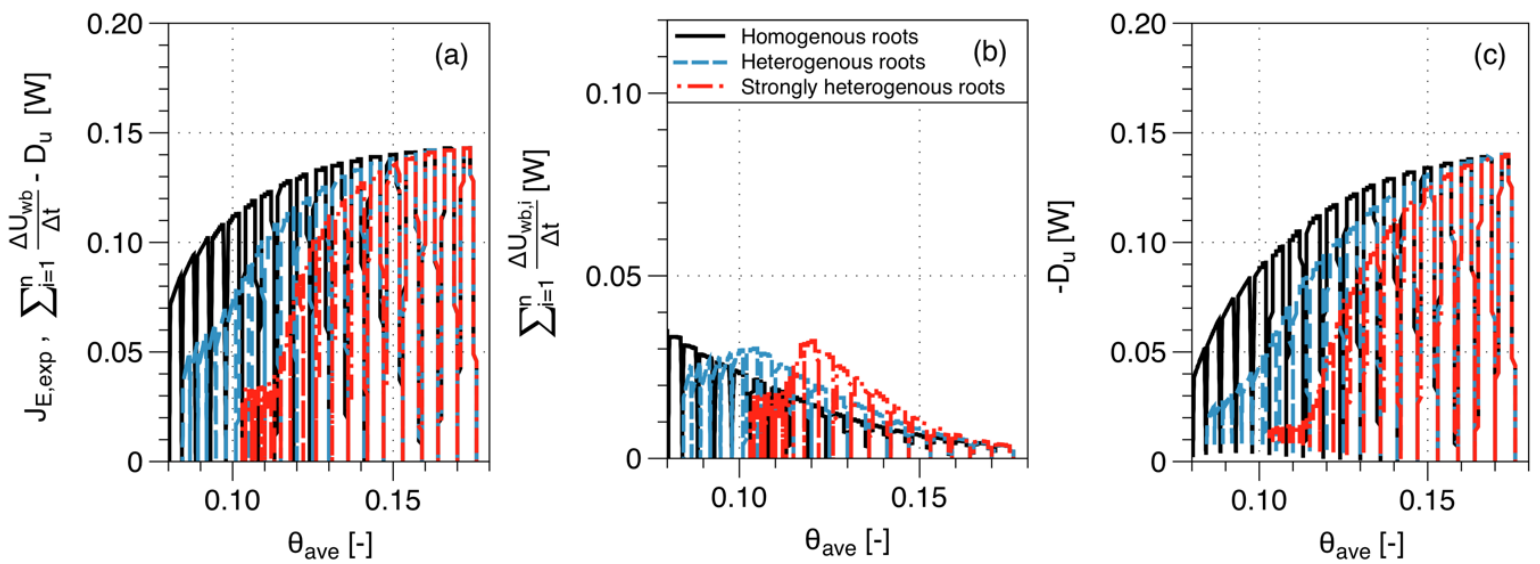

Fig S02: Exported energy and its components for the soil-plant-system over the course of a drying experiment and different root water uptake scenarios. Results from a transient model. As in Fig. S01(b), the time axis was replaced by the average soil water content. (a) Total energy exported from the system at the root collar. It is the sum of the two components given in the other subplots, (b) Component due to change in soil binding energy, which is due to both soil drying and enhanced heterogeneity (compare Fig. S02b), (c) Component due to energy dissipation by water flow from the soil into the root. 


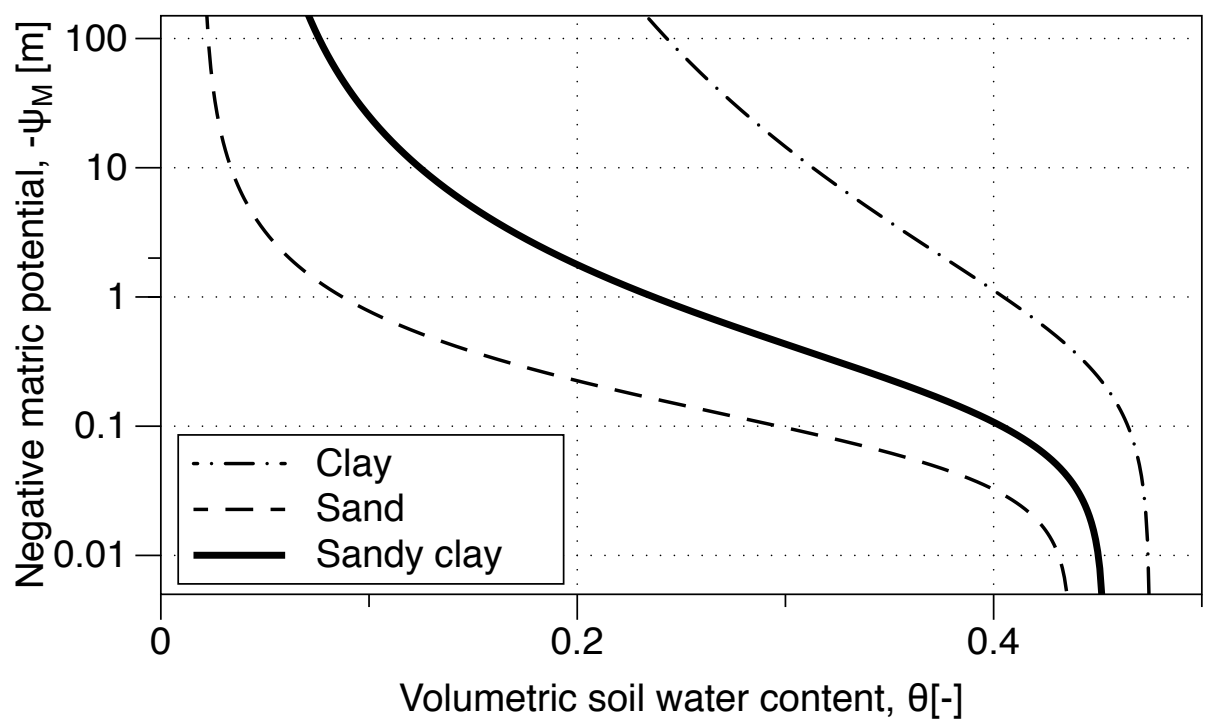

Fig S03: Soil water retention curve for the parameterizations indicated in Table 1 . The heavy line indicates the parameterization used in the main text and model results are given in the manuscript. The light lines indicate the extremes of the water retention spectrum. Results obtained with those soil parameterizations, but otherwise same scenarios as in given in the main text, are given in Figs S04 and S05 (sand) and Figs S06 and S07 (clay). 

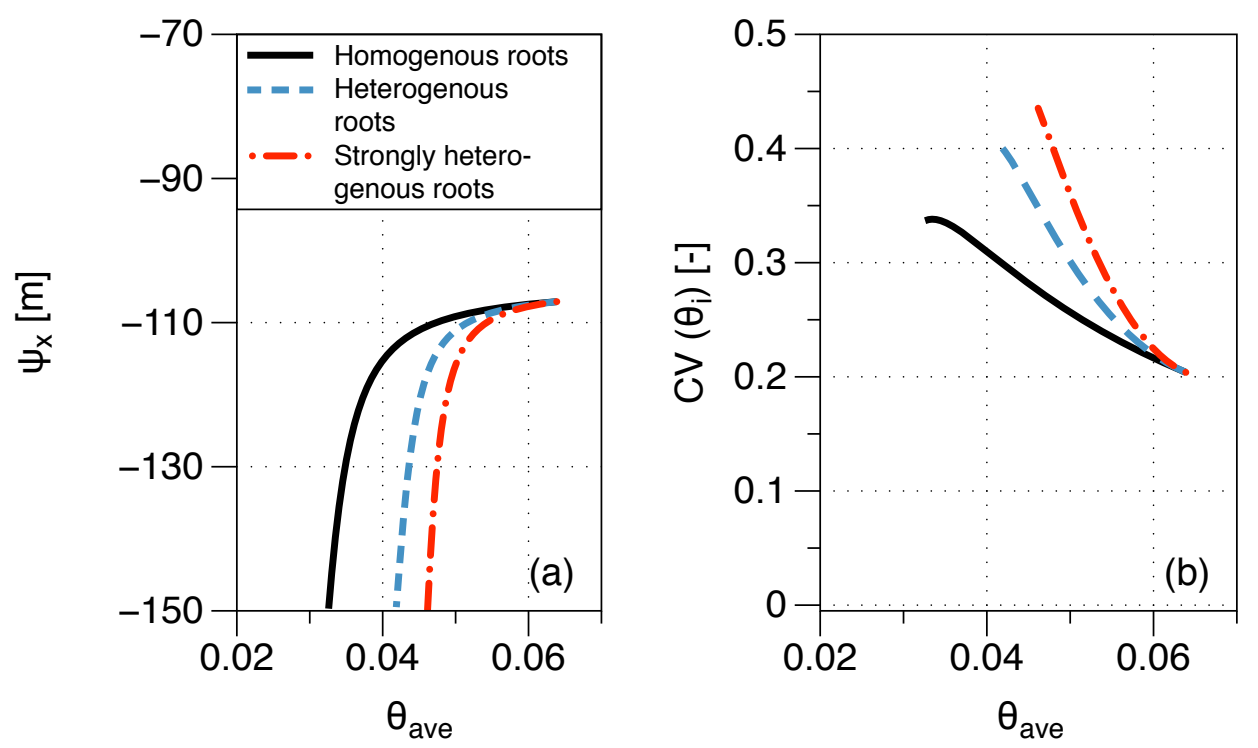

Fig S04: Model results for the simple model for a soil with low water retention (sand, see Table S01 for parameters), otherwise corresponding to Fig. 3 in the main text. (a) Evolution of the xylem water potential over the course of root water uptake, (b) Evolution of the coefficient of variation of soil water content during the simulation.
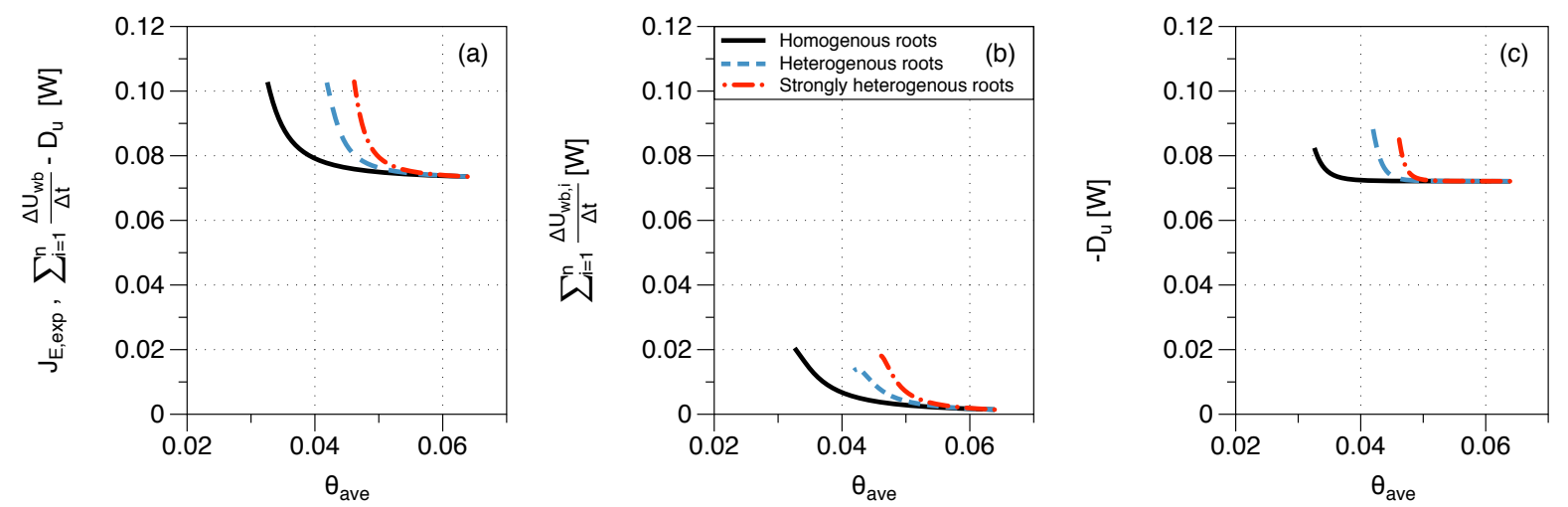

Fig S05: Exported energy and its components for a soil with low water retention (sand, see Table S01 for parameters), otherwise corresponding to Fig. 4 in the main text. (a) Total energy exported from the system at the root collar. (b) Component due to the change in soil binding energy, (c) Component due to dissipation by water flow from the soil into the root. 

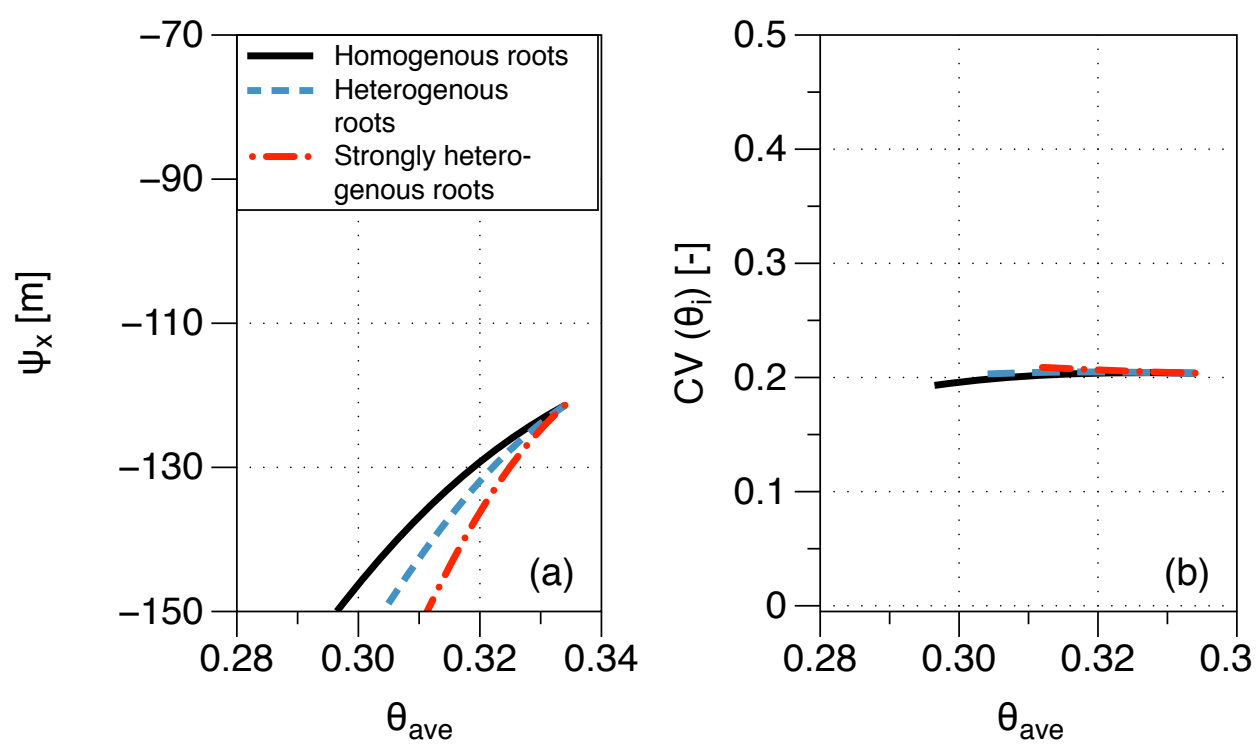

Fig S06: Model results for the simple model for a soil with high water retention (clay, see Table S01 for parameters), otherwise corresponding to Fig. 3 in the main text. (a) Evolution of the xylem water potential over the course of root water uptake, (b) Evolution of the coefficient of variation of soil water content during the simulation.
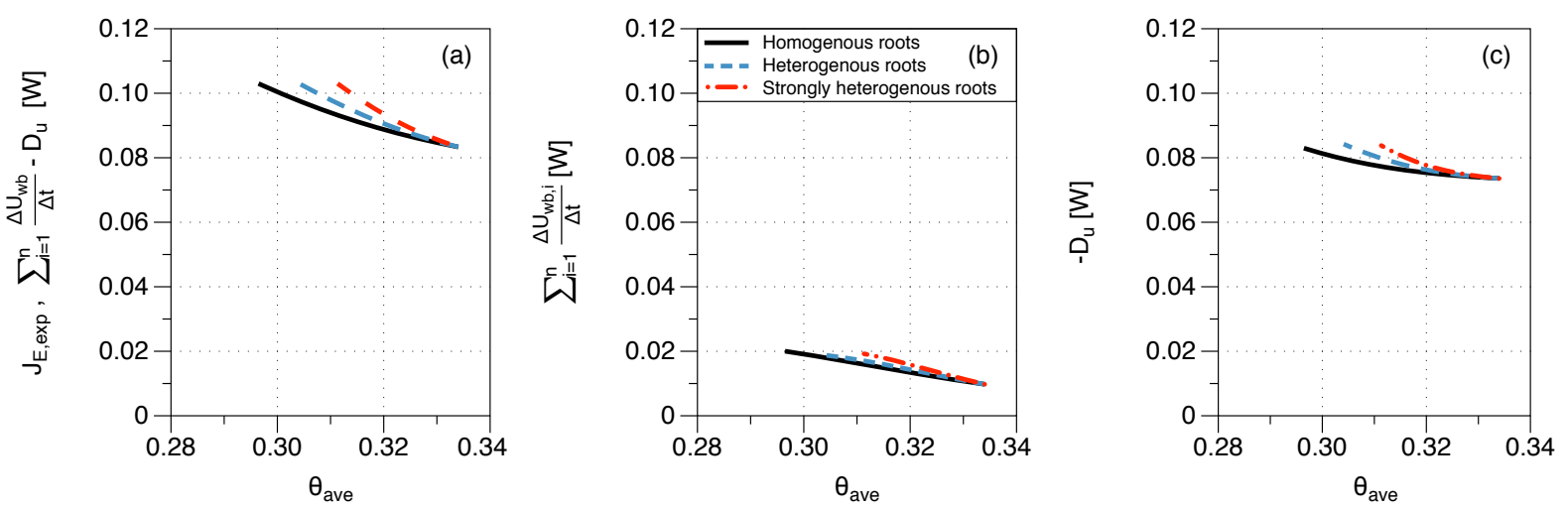

Fig S07: Exported energy and its components for a soil with high water retention (clay, see Table S01 for parameters), otherwise corresponding to Fig. 4 in the main text. (a) Total energy exported from the system at the root collar, (b) Component due to the change in soil binding energy, (c) Component due to dissipation by water flow from the soil into the root. 


\section{References}

Rawls, W. J., Ahuja, L. A., Brakensiek, D. L., and Shirmohammadi, A.: Infiltration and soil water movement, in: Handbook of Hydrology, edited by Maidment, D. R., Chap. 5, pp. 5.15.51, MacGraw-Hill, New York, 1993.

van Genuchten, M. T.: A closed-form equation for predicting the hydraulic conductivity of unsaturated soils, Soil Science Society of America Journal, 44, 892-898,

doi:10.2136/sssaj1980. 03615995004400050002x, 1980. 